\title{
Tecnologias Educacionais: um Estudo Prospectivo
}

\author{
Educational Technologies: a Prospective Study
}

\author{
Flávio Santos da Silva ${ }^{1}$ \\ Leyla Carolyne da Silva Santos ${ }^{1}$ \\ Ibsen Mateus Bittencourt ${ }^{1}$ \\ Sílvia Beatriz Uchôa ${ }^{1}$ \\ Tatiane Luciano Balliano ${ }^{1}$ \\ ${ }^{1}$ Universidade Federal de Alagoas, Maceió, AL, Brasil
}

\begin{abstract}
Resumo
O presente estudo tem como metodologia uma análise prospectiva das tecnologias educacionais, considerando as patentes do repositório internacional World Intellectual Property Organization (WIPO), as patentes nacionais presentes no Instituto Nacional de Propriedade Industrial (INPI) e em trabalhos na literatura referente ao tema. Com base no levantamento feito definiu-se as principais vertentes da educação em que essas tecnologias estão presentes e sua expectativa de evolução. Esse estudo também analisa o posicionamento dos principais países na utilização de tais tecnologias e as empresas que despontam como maiores depositantes de patentes e sua evolução temporal. Descobriu-se por meio dessa análise que as tecnologias educacionais possuem forte investimento dos países melhores colocados em rankings internacionais de educação e que o Brasil está aquém do que é produzido nesses países. Assim, este estudo apresenta como resultados o fato de que tecnologias educacionais estão diretamente relacionadas ao investimento que o país faz em educação e como a fonte desses investimentos impacta na pesquisa de novas tecnologias educacionais.
\end{abstract}

Palavras-chave: Educacional. Tecnologia. Prospecção.

\begin{abstract}
The present study has a methodology a prospective analysis of the educational technologies considering the patents of the World Intellectual Property Organization (WIPO), Instituto Nacional de Propriedade Industrial (INPI) and works in the literature related to the subject. Based on the survey, we defined the main aspects of education in which these technologies are present and their expectation of evolution. This study also analyzes the position of the countries in the use of such technologies, as well as the companies that emerge as major depositors of patents and their temporal evolution. It was discovered that the educational technologies have a strong investment of the countries best placed in international education rankings and that Brazil falls short of what is produced in these countries. Thus, this study resulted in educational programs in progress related to the country's investment in education and as a source of investments that impact on the research of new educational technologies.
\end{abstract}

Keywords: Educational. Technology. Prospection.

Área Tecnológica: Educação. Tecnologia da Informação e Comunicação. 


\section{Introdução}

Tecnologia educacional consiste no uso efetivo de ferramentas tecnológicas na aprendizagem que, endossada pela Academia BrianCert (2015), trata-se de uma série de ferramentas, como mídia, máquinas, e hardware de rede, e de perspectivas teóricas subjacentes para sua aplicação efetiva, pois incluem diversos tipos de mídias como texto, áudio, imagens, animação, transmissão de vídeo, aplicativos, aprendizado baseado em computador, intranet, internet $e$ aprendizagem baseada na web.

Tais tecnologias dividem-se, como indica a Academia BrianCert (2015), em três tipos: síncrona e assíncrona, aprendizado linear, aprendizado colaborativo; e nas seguintes classificações (DEY, 2017, p. 22): impressão visual (verbal) ou duplicada, visual não projetada bidimensional, visual não projetada tridimensional, visual projetada, áudio, audiovisual projetada com movimento (multissensorial), pacotes multimídia, mídias emergentes.

Discutir sobre a evolução das tecnologias educacionais, conforme apontam Mishra, Koehler e Kereluik (2009), tem considerável importância devido à rápida mudança de tecnologias e pelo papel que elas assumem na educação. Além disso, por meio da análise de patentes nesse contexto, será possível verificar os países que despontam no desenvolvimento dessas tecnologias e realizar uma análise comparativa com os países que possuem os maiores índices em ciência, leitura e matemática, segundo dados levantados pelo Programa Internacional de Avaliação de Alunos (PISA).

Assim, este trabalho tem por objetivo caracterizar os principais segmentos em que as tecnologias educacionais são aplicadas, bem como os países que mais investem no desenvolvimento dessas tecnologias, a perspectiva de crescimento das tecnologias educacionais e o que o investimento nesse tipo de tecnologia pode indicar sobre determinado país. A seção de metodologia apresentará como os dados foram levantados, onde tais dados foram coletados e como ocorreu a mineração desses dados para gerar os resultados apresentados neste trabalho. A seção de resultados e discussão apresenta o que foi retornado nas buscas e faz uma sucinta discussão comparando o que foi retornado com as ocorrências nas diversas bases de dados e com informações de bases internacionais como o PISA. A seção de conclusão contém uma análise da maturidade dessas tecnologias e indicações de trabalhos futuros relacionados ao tema.

\section{Metodologia}

Neste trabalho foi realizada uma revisão da literatura científica na base WIPO e INPI para busca de patentes relacionadas ao tema. Também foram realizadas pesquisas na literatura em repositórios como Scielo, Google Acadêmico e Periódicos Capes. A definição das palavras chave a serem utilizadas para pesquisa nas bases de patentes seguiu três parâmetros:

a) Palavras-chave com base nos segmentos da educação em que as tecnologias educacionais atuam apontados por Cardoso, Santos e Gatti (2015, p. 392).

b) Palavras-chave com base nos tipos de tecnologias educacionais apontados por Brian Cert (2015).

c) Palavras-chave com base nas classificações das tecnologias educacionais (DEY, 2017, p. 22). 
No Quadro 1, as palavras-chave presentes na coluna à esquerda foram as utilizadas nas bases internacionais. Por conseguinte, na coluna à direita listadas as palavras-chave utilizadas na base de patentes Instituto Nacional de Propriedade Industrial (INPI).

Quadro 1 - Palavras-chave utilizadas nas pesquisas de patentes

\begin{tabular}{|c|c|}
\hline PALAVRAS-CHAVE BASES INTERNACIONAIS & PALAVRAS-CHAVE BASE NACIONAL \\
\hline $\begin{array}{l}\text { education, technology, evaluation, game, development, } \\
\text { simulation, EAD, synchronous, asynchronous, linear, } \\
\text { collaborative, visual, two-dimensional, audio, three- } \\
\text { dimensional, audio-visual, multi-media, media. }\end{array}$ & $\begin{array}{l}\text { educação, tecnologia, avaliação, jogo, } \\
\text { desenvolvimento, simulação, EAD, síncrona, } \\
\text { assíncrona, linear, colaborativo, visual, bidimensional, } \\
\text { tridimensional, áudio, audiovisual, multimídia, mídia. }\end{array}$ \\
\hline
\end{tabular}

Fonte: Elaborado pelos autores deste artigo

O Quadro 2 apresenta os cruzamentos realizados entre essas palavras-chave e os operadores, contudo, como cada base possui uma padronização diferente para os operadores, eles foram generalizados.

Quadro 2 - Cruzamentos de palavras-chave e operadores utilizados nas pesquisas de patentes

\begin{tabular}{|c|c|}
\hline CRUZAMENTOS BASES INTERNACIONAIS & CRUZAMENTOS BASE NACIONAL \\
\hline $\begin{array}{c}\text { (education* and technolog*) } \\
\text { (education* and technolog* and evaluation) } \\
\text { (education* and technolog* and gam*) } \\
\text { (education* and technolog* and development) } \\
\text { (education* and technolog* and simulat*) } \\
\text { (education* and technolog* and ead) } \\
\text { (education* and technolog* and } \\
\text { (synchronous or asysnchronous)) } \\
\text { (education* and technolog* and linear) } \\
\text { (education* and technolog* and collaborative) } \\
\text { (education* and technolog* and visual) } \\
\text { (education* and technolog* and two-dimensional) } \\
\text { (education* and technolog* and three-dimensional) } \\
\text { (education* and technolog* and audio) } \\
\text { (education* and technolog* and audio-visual) } \\
\text { (education* and technolog* and } \\
\text { (media or multi-media)) }\end{array}$ & $\begin{array}{c}\text { (educa* and tecnolog*) } \\
\text { (educa* and tecnolog* and avalia*) } \\
\text { (educa* and tecnolog* and jogo) } \\
(\text { educa* and tecnolog* and desenvolv*) } \\
\text { (educa* and tecnolog* and simula*) } \\
\text { (educa* and tecnolog* and ead) } \\
\text { (educa* and tecnolog* and (s?ncrono or ass?ncrono)) } \\
\text { (educa* and tecnolog* and linear) } \\
\text { (educa* and tecnolog* and colaborativo) } \\
\text { (educa* and tecnolog* and visual) } \\
\text { (educa* and tecnolog* and bidimensional } \\
\text { or (duas and dimens?es)) } \\
\text { (educa* and tecnolog* and audio) } \\
\text { (educa* and tecnolog* and ?udio?visual) } \\
\text { (educa* and tecnolog* and (m?dia or multi?m?dia)) }\end{array}$ \\
\hline
\end{tabular}

Fonte: Elaborado pelos autores deste artigo

Os conteúdos retornados nas bases foram tabulados e minerados em um editor de planilhas eletrônicas, e foram também utilizados para a criação dos gráficos presentes neste trabalho.

\section{Resultados e Discussão}

As pesquisas pelo termo mais universal do tema na base WIPO por meio do cruzamento (education* and technolog*) mostraram os países indicados na Tabela 1. 
Tabela 1 - Maiores países depositantes de patentes para o cruzamento (education* and technolog*) na base WIPO

\begin{tabular}{cc} 
DePositante & Patentes DePositadas \\
China & 3717 \\
PCT & 776 \\
United States & 693 \\
Republic of Korea & 669 \\
India & 169 \\
European Patent Office & 130 \\
Japan & 109 \\
Australia & 79 \\
Canada & 47 \\
Russian Federation & 41 \\
\hline
\end{tabular}

Fonte: Base World Intellectual Property Organization (2018)

Comparando os dados da Tabela 1 com as informações disponibilizadas pelo PISA 2015 RESULTS (2018), presentes nas Figuras 1, 2 e 3 que tratam do desempenho dos estudantes em ciências, leitura e matemática nos diferentes países. Percebe-se que a China, país que mais deposita patentes relacionadas às tecnologias educacionais, figura entre os 10 países mais bem ranqueados pelo PISA 2015 RESULTS (2018) nas três categorias, e que países como Coreia, Japão, Canadá e alguns países europeus também aparecem nas primeiras posições.

Nesse contexto, a China merece considerável destaque uma vez que possui mais que o dobro de patentes registradas e está entre os melhores desempenhos educacionais do mundo em ciências, leitura e matemática. O desempenho do país está diretamente relacionado à qualidade da sua educação, conforme indicado no relatório da Organisation for Economic Co-operation and Development (2016), elaborado especificamente para a China e que apresenta dados como a origem do fundo de investimento em educação no país em que $80 \%$ dos investimentos são é originários do Governo e o restante de iniciativas privadas.

Figura 1 - Países com os melhores desempenhos no PISA 2015 RESULTS (2018) para a categoria ciências

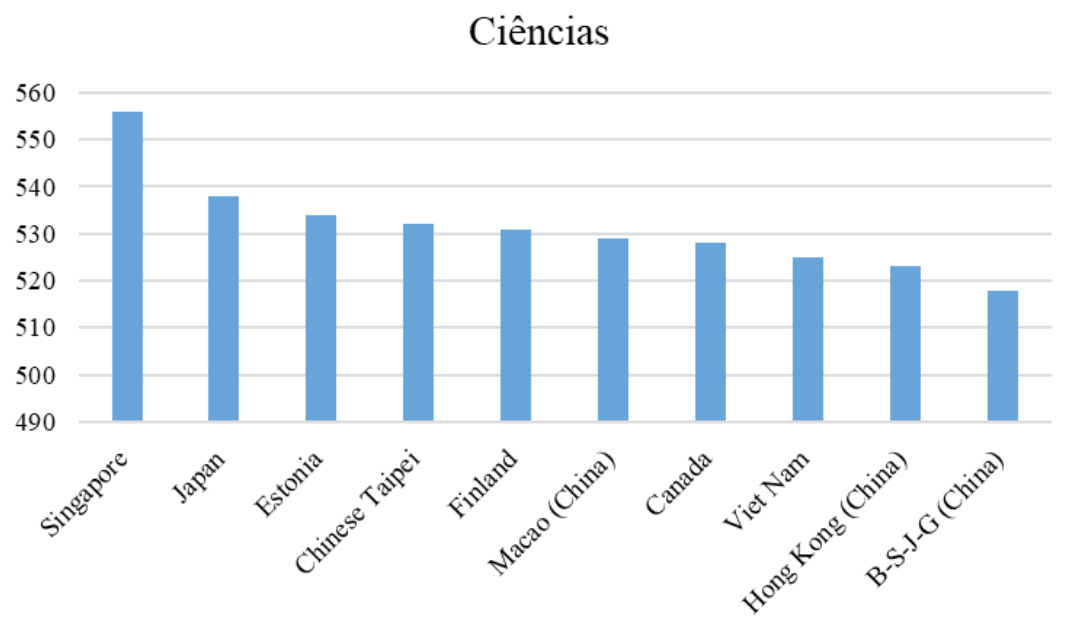

Fonte: PISA (2015) 
Figura 2 - Países com os melhores desempenhos no PISA 2015 RESULTS (2018) para a categoria leitura

\section{Leitura}

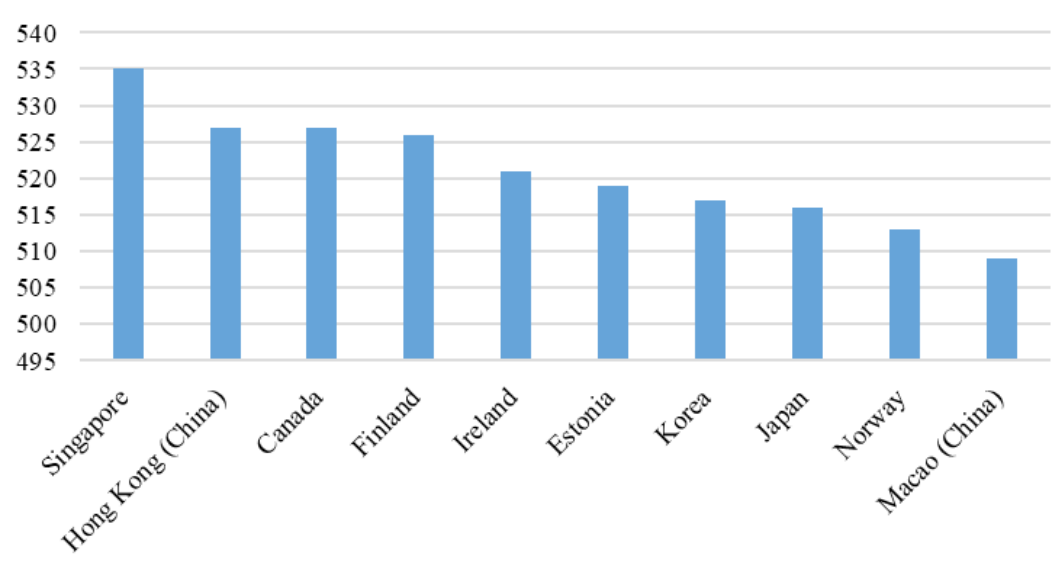

Fonte: PISA (2015)

Figura 3 - Países com os melhores desempenhos no PISA 2015 RESULTS (2018) para a categoria matemática

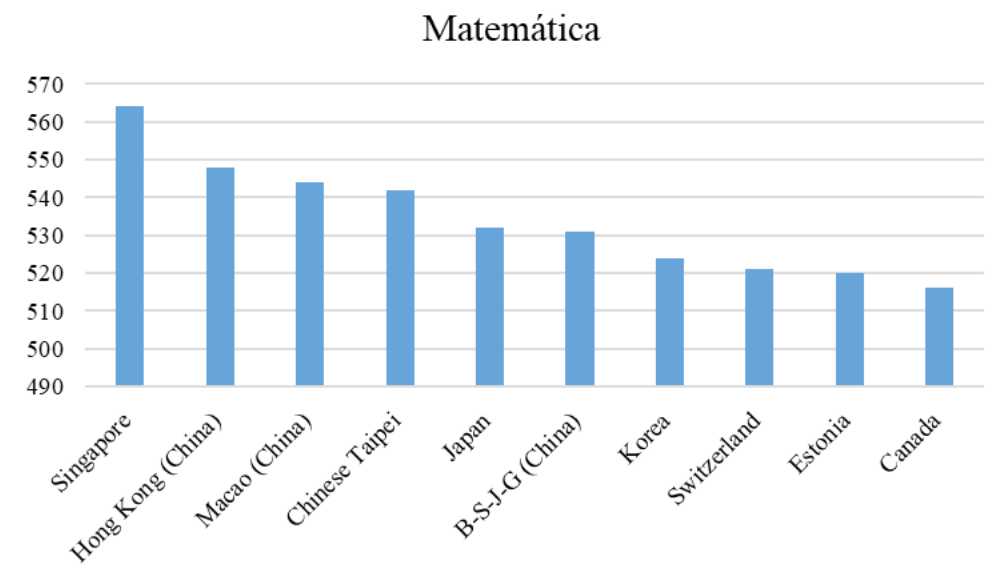

Fonte: PISA 2015 RESULTS (2018)

Ao minerar na base WIPO por patentes diretamente relacionadas aos segmentos de estudos de tecnologias educacionais presentes na literatura (CARDOSO; SANTOS; GATTI, 2015, p. 392), os resultados são como estes visualizados na Tabela 1.

Tabela 1 - Maiores depósitos de patentes para as strings criadas a partir do levantamento feito por Cardoso, Santos e Gatti (2015, p. 392)

$\begin{array}{cc}\text { STRING DE BUSCA } & \text { PATENTES } \\ \text { (education* and technolog* and development) } & 394 \\ \text { (education* and technolog* and simulat*) } & 217 \\ \text { (education* and technolog* and gam*) } & 169 \\ \text { (education* and technolog* and evaluation) } & 147\end{array}$

Fonte: Base World Intellectual Property Organization (2018) 
Analisando os dados da Tabela 1 e comparando-os com o levantado por Cardoso, Santos e Gatti (2015, p. 392) para a distribuição dos tipos de trabalhos acadêmicos relacionados às tecnologias educacionais, percebe-se que recursos relacionados ao desenvolvimento são, em ambos os casos, bastante focados quando se pensa tanto na produção acadêmica, quanto na produção de produtos/serviços. Todavia, ainda que estudos relacionados a tecnologias educacionais e games ocupem o primeiro lugar em trabalhos acadêmicos (CARDOSO; SANTOS; GATTI, 2015), no registro de patentes, tal categoria ocupa somente a terceira colocação. Isso deve-se, provavelmente, ao fato de que o registro do software é opcional uma vez que ele é protegido por copyrights (TIGRE; MARQUES, 2009), tornando a quantidade de patentes inferior ao esperado.

Ao pesquisar pelos cruzamentos inerentes aos tipos de tecnologias educacionais apontados pela Academia BrianCert (2015), os resultados gerados foram os visualizados na Tabela 2.

Ao observar esses dados, percebe-se essa categorização para as tecnologias educacionais é pouco utilizada pelos depositantes de patentes, pois do contrário o tipo que diz respeito à tecnologia educacional colaborativa teria muito mais registros encontrados, porque trata-se de um dos principais tipos de tecnologias educacionais atualmente criadas.

Tabela 2 - Patentes encontradas na base WIPO para os tipos de tecnologias educacionais

\begin{tabular}{cc} 
STRING DE BUSCA & PATENTES \\
(education* and technolog* and (synchronous or asysnchronous)) & 58 \\
(education* and technolog* and linear) & 51 \\
(education* and technolog* and collaborative) & 12 \\
\hline
\end{tabular}

Fonte: Base World Intellectual Property Organization (2018)

Buscando pelas strings relacionadas às classificações das tecnologias educacionais (DEY, 2017, p. 22), foram encontradas as patentes listadas na Tabela 3.

Tabela 3 - Patentes encontradas na base WIPO para as classificações de tecnologias educacionais

$\begin{array}{cc}\text { STRING DE Busca } & \text { PATENTES } \\ \text { (education* and technolog* and audio) } & 261 \\ \text { (education* and technolog* and visual) } & 178 \\ \text { (education* and technolog* and (media or multi-media)) } & 152 \\ \text { (education* and technolog* and three-dimensional) } & 123 \\ \text { (education* and technolog* and audio-visual) } & 63 \\ \text { (education* and technolog* and two-dimensional) } & 60\end{array}$

Fonte: Base World Intellectual Property Organization (2018)

Analisando os resultados da Tabela 2, é possível observar que poucos depositantes utilizam no depósito de suas patentes a categorização de tipos utilizada na literatura. Isso ocorre porque os depositantes acabam priorizando por tipos mais genéricos ou com uma padronização internacionalizada, como a Classificação Internacional de Patentes (CIP) cujos dados são apresentados no gráfico da Figura 5 mais à frente. 
Com os resultados da Tabela 3 percebe-se que as tecnologias educacionais que utilizam recursos de áudio ou visuais são dominantes nos depósitos de patentes. Ao varrer tais números em busca da tendência de estudos e de depósitos de patentes para os próximos anos nota-se que há um crescimento exponencial em depósitos de patentes relacionadas a áudio, visual $e$ tridimensional, conforme apresentado na Figura 4.

Figura 4 - Crescimento dos depósitos de patentes por classificações de tecnologias educacionais de 2013 a 2017

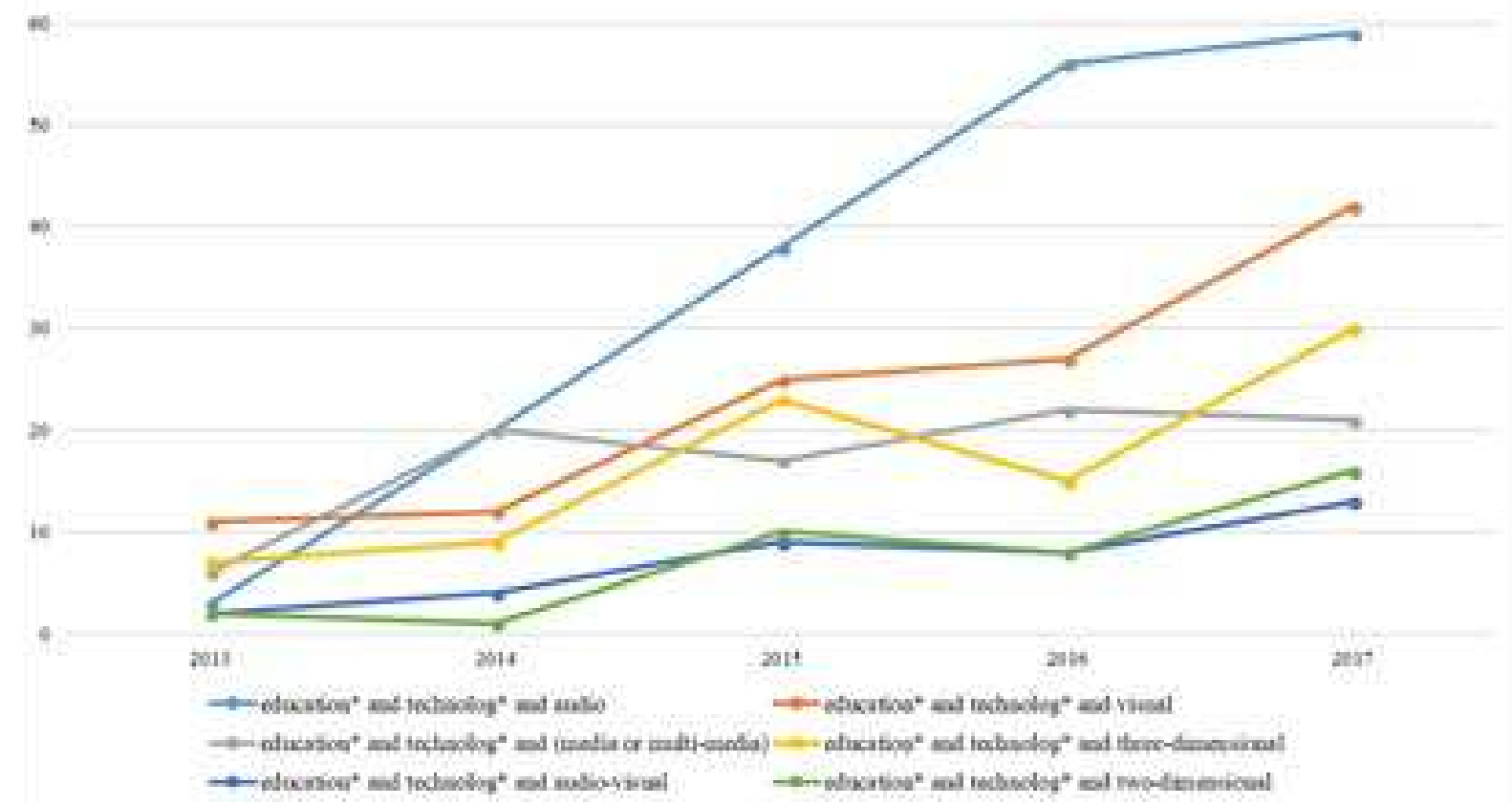

Fonte: World Intellectual Property Organization (2018)

Tal crescimento nessas três categorias deve-se pelo acelerado aumento na disponibilidade de equipamentos que fazem uso de recursos de áudio, vídeo e três dimensões como celulares e tablets (HEATHER et al., 2016). Como tais equipamentos se tornaram bastante comuns $e$ acessíveis ao público em geral e possuem uma estimativa de crescimento ano a ano pelo menos até 2025 (HEATHER et al., 2016), então estima-se que os dados apresentados na Figura 4 mantenham a tendência com tecnologias educacionais que utilizam recursos de áudio, vídeo $e$ três dimensões sendo o foco das patentes.

O gráfico da Figura 5 apresenta as famílias das patentes depositadas para tecnologias educacionais, de acordo com a CIP. 
Figura 5 - Distribuição das patentes por categoria CIP utilizando o cruzamento (education* and technolog*)

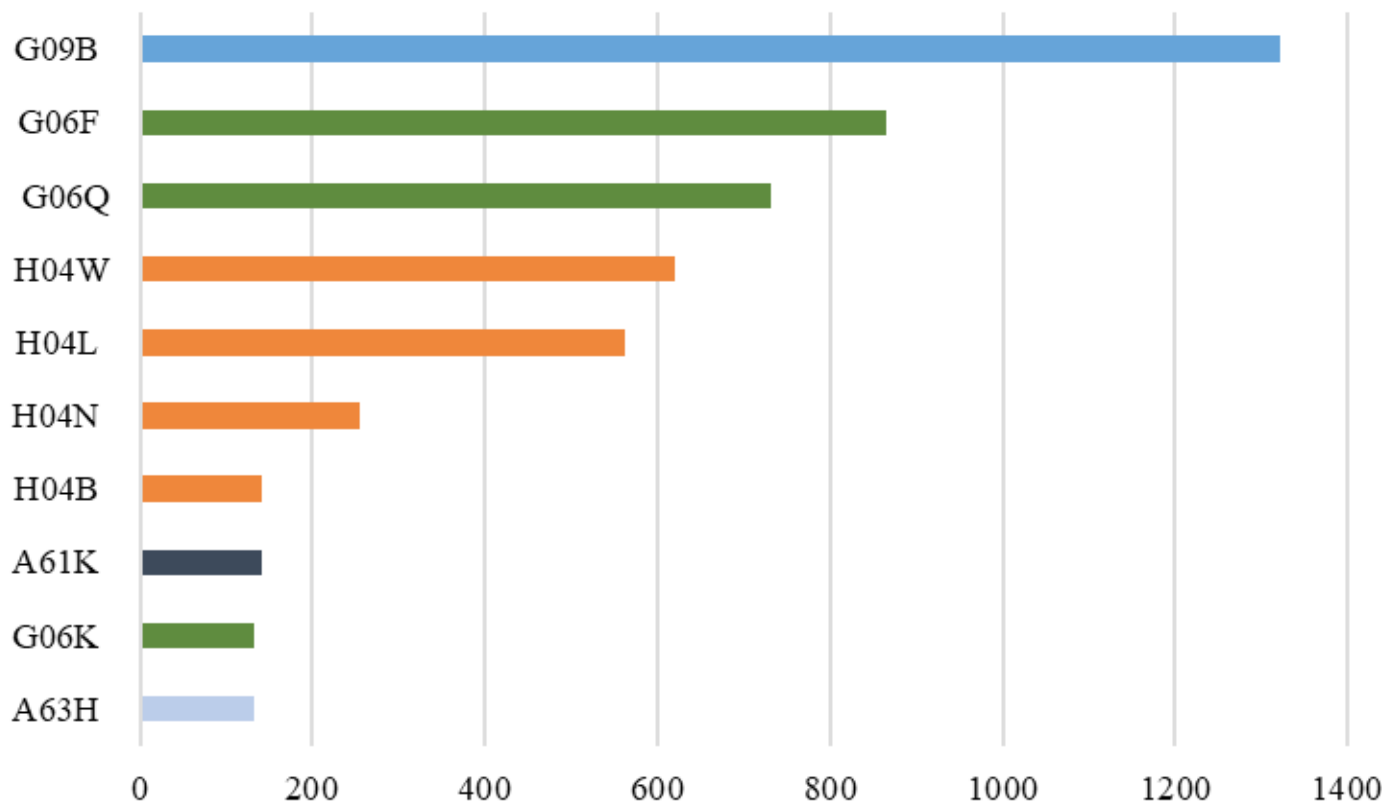

Fonte: Base World Intellectual Property Organization (2018)

De acordo com os dados do gráfico da Figura 5, observa-se que as famílias mais utilizadas nas patentes depositadas para tecnologias educacionais são as da categoria G09 e G06. Essas categorias equivalem, respectivamente, a patentes relacionada à educação, à criptografia, à apresentação visual, aos anúncios, aos logotipos e ao cômputo, ao cálculo, à contagem. Ao considerar a CIP completa, as patentes estão massivamente concentradas em aparelhos educativos ou de demonstração e simuladores (WORLD INTELLECTUAL PROPERTY ORGANIZATION, 2018).

Ainda em relação as buscas realizadas para a string (education* and technolog*), as organizações que mais depositam patentes são as visualizadas na Tabela 7 a seguir.

Tabela 4 - Maiores depositantes de patentes na base WIPO para o cruzamento (education* and technolog*)

\begin{tabular}{cc} 
DePositante & PateNTES \\
Samsung Electronics Co., LTD. & 735 \\
Dianjing University of Technology and Education & 492 \\
한국기술교육대학교 산학협력단 & 349 \\
Korea University of Technology and Education Industry- & 382 \\
University Cooperation Foundation & 159 \\
\hline
\end{tabular}

Fonte: Base World Intellectual Property Organization (2018)

Os dados da Tabela 4 apresentam uma surpresa na primeira colocação, uma vez que todas as demais depositantes são organizações com fins educacionais. Contudo, ao analisar as políticas educacionais adotadas pela empresa (SAMSUNG, 2018), percebe-se que essa empresa 
tem realizado um investimento considerável em tecnologias educacionais e que suas invenções possuem bastante apelo para uso na educação. Tal constatação passa a fazer muito sentido quando é levado em consideração que a empresa tem sua base de atuação em países asiáticos e, conforme já visualizado, os países asiáticos possuem resultados acima da média no desenvolvimento educacional de seu povo (PISA, 2015). As outras colocações da Tabela 4 também são ocupadas por instituições asiáticas, o que endossa a afirmação de que os investimentos em tecnologias educacionais estão diretamente relacionados ao desempenho estudantil no PISA 2015 RESULTS (2018).

Ao realizar as buscas na base nacional de patentes, utilizando as strings citadas no Quadro 2 , tem-se os resultados apresentados na Tabela 5.

Tabela 5 - Resultados de patentes buscadas na base INPI

\begin{tabular}{|c|c|}
\hline CRUzamentos base NACIONAL & Patentes depositadas \\
\hline (educa* and tecnolog*) & 36 \\
\hline (educa* and tecnolog* and avalia*) & 2 \\
\hline (educa* and tecnolog* and jogo) & 2 \\
\hline (educa* and tecnolog* and desenvolv*) & 9 \\
\hline (educa* and tecnolog* and simula*) & 3 \\
\hline (educa* and tecnolog* and ead) & 0 \\
\hline $\begin{array}{l}\text { (educa* and tecnolog* and } \\
\text { (s?ncrono or ass?ncrono)) }\end{array}$ & 0 \\
\hline (educa* and tecnolog* and linear) & 0 \\
\hline (educa* and tecnolog* and colaborativo) & 0 \\
\hline (educa* and tecnolog* and visual) & 1 \\
\hline $\begin{array}{l}\text { (educa* and tecnolog* and bidimensional } \\
\text { or (duas and dimens?es)) }\end{array}$ & 0 \\
\hline (educa* and tecnolog* and audio) & 4 \\
\hline (educa* and tecnolog* and ?udio?visual) & 1 \\
\hline (educa* and tecnolog* and (m?dia or multi?m?dia)) & 0 \\
\hline
\end{tabular}

Fonte: Base INPI (2018)

As patentes registradas no Brasil para tecnologias educacionais, no contexto apresentado na introdução, ainda são escassas como visualizado anteriormente. Apesar disso, as que estão presentes na base INPI seguem as tendências das patentes presentes na base WIPO, como: a baixa utilização dos tipos de tecnologias educacionais ao descrever as patentes dessa temática; e a maior quantidade de patentes relacionadas a tecnologias educacionais que utilizam recursos de áudio, visuais ou ambos.

As buscas na base INPI por família CIP indicam que o país tem seguido a tendência mundial e direcionado suas pesquisas para temas associados à família G e H, conforme mostra Figura 6. 
Figura 6 - Distribuição das patentes por categoria CIP utilizando o cruzamento (educa* and tecno$\log *)$

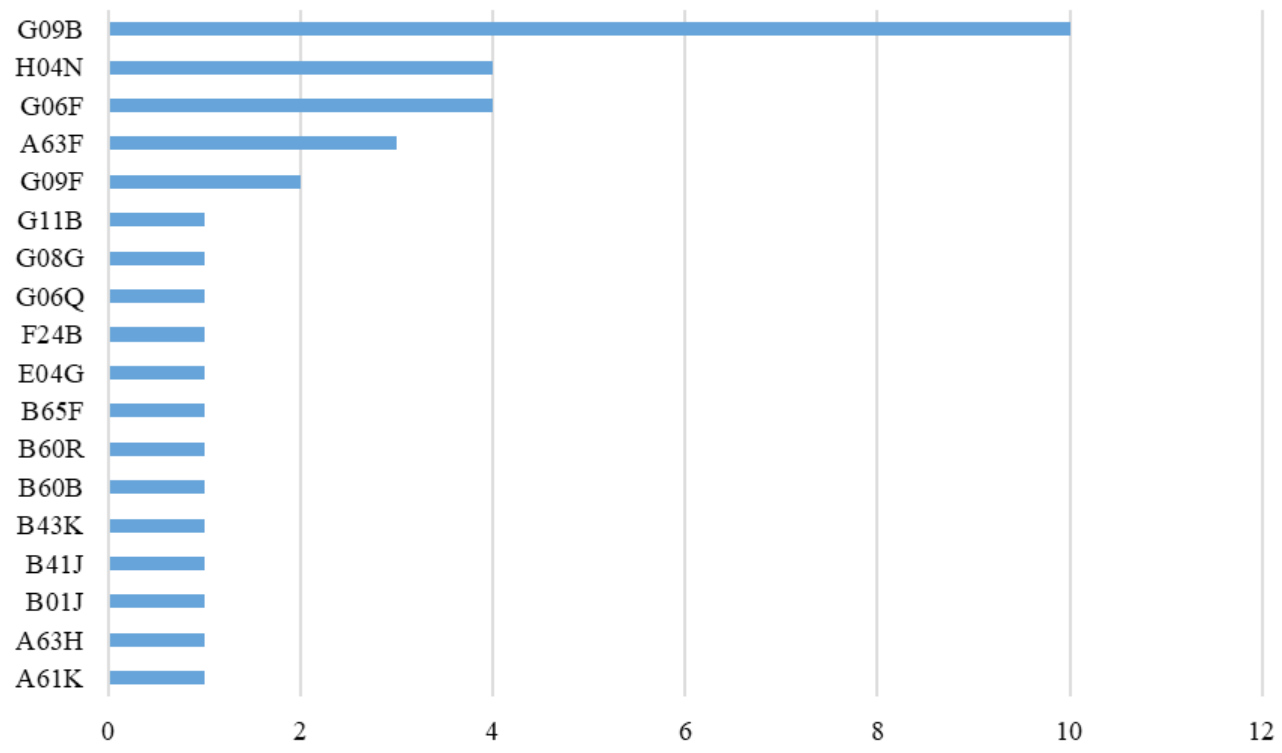

Fonte: Base INPI (2018)

É perceptível que a baixa quantidade de patentes relacionadas às tecnologias educacionais no Brasil possui relação com sua posição no PISA 2015 RESULTS (2018), já que o país figura entre os piores resultados, inclusive abaixo da média internacional. Além disso, boa parte do investimento no desenvolvimento da educação no país vem do Governo e como os gastos com investimento em educação foram congelados no país por 20 anos (AMORIM, 2016), espera-se que o cenário das tecnologias educacionais não sofra alteração durante o mesmo período. Cabe salientar também que outro fator que impede o crescimento das tecnologias educacionais é o elevado custo que a tecnologia em geral possui no Brasil (PRIOR, 2011).

Assim, de acordo com as patentes e com a literatura pesquisada, é possível prospectar que a evolução das tecnologias educacionais acompanhará o desenvolvimento educacional de países que melhor figuram no PISA 2015 RESULTS (2018) e que tais tecnologias estão condicionadas ao progresso de equipamentos eletrônicos. Estima-se ainda que as tecnologias educacionais dos próximos anos comecem a convergir para soluções educacionais voltadas para o aprendizado social em nuvem, uma vez que é para esse caminho que a computação está convergindo (BRITLAND, 2013).

\section{Considerações Finais}

Analisando a literatura disponível e as patentes registradas e concedidas, é possível perceber que nos países com melhores índices na educação as tecnologias educacionais são uma importante vertente de pesquisas, não somente por parte de instituições de ensino, mas também por parte de organizações privadas. As pesquisas atuais nessas tecnologias estão fortemente enlaçadas a recursos de áudio, visuais ou ambos que utilizem equipamentos de uso cotidiano da população e de fácil aquisição para o ambiente escolar.

A tendência de evolução das tecnologias educacionais está relacionada ao crescimento de equipamentos como celulares e tablets e os maiores investidores nessa tecnologia são os países 
asiáticos cujos índices educacionais são altíssimos. Nesse sentido, o Brasil acaba se tornando um país que ainda está em fase inicial de desenvolvimento de tais tecnologias quando comparado ao que tem sido produzido em países como China e Coreia.

Assim, com essa prospecção foi possível perceber que os segmentos em que as tecnologias educacionais mais atuam são os de áudio e visual para três dimensões. Os países que mais investem nesses recursos são em sua maioria países asiáticos e que ocupam as melhores colocações em rankings internacionais de classificação do desenvolvimento estudantil, como o PISA. Percebeu-se também que a perspectiva de crescimento dessas tecnologias está diretamente relacionada com a evolução de equipamentos eletrônicos, como smartphones e que o investimento em tecnologias educacionais é diretamente proporcional à qualidade do ensino $e$ do investimento governamental e privado na educação de um país.

Como melhoria para trabalhos futuros, sugere-se uma ampliação da base de dados pesquisada, englobando repositórios que não foram considerados no trabalho em questão. Faz-se necessário um maior cruzamento entre as palavras-chave e a inserção de novas palavras para maior enriquecimento dos resultados produzidos e uma análise comparativa mais ampla entre a evolução de recursos eletrônicos/computacionais e as tecnologias educacionais.

\section{Referências}

ACADEMIA BRIANCERT. 3 Different Types of Educational Technology. BrianCert Academy. Education \& Technology. November, 2015. Disponível em: <https://www.braincert.com/ blogs/9education-technology/119-3-different-types-of-edu\%D1\%81\%D0\%B0t\%D1\%96\%D0\%BEnaltechnology >. Acesso em: 21 jun. 2018.

AMORIM, Felipe. PEC do teto é aprovada em votação final e congela gastos por 20 anos. UOL notícias Política. Brasília, DF, 2016. Disponível em: <https://noticias.uol.com.br/politica/ultimasnoticias/2016/12/13/pec-que-congela-gastos-do-governo-por-20-anos-e-aprovada-em-votacao-final. htm >. Acesso em: 21 jun. 2018.

BRITLAND, Matt. What is the future of technology in education. The Guardian, 2013. Disponivel em: <https://www.theguardian.com/teacher-network/teacher-blog/2013/jun/19/technology-futureeducation-cloud-social-learning > . Acesso em: 21 jun. 2018.

CARDOSO, Rogério; SANTOS, Otávio Ventura dos; GATTI, Daniel Couto. Revisão sistemática de objetos de aprendizagem para o ensino de computação. In: X CONFERÊNCIA LATINOAMERICANA DE OBJETOS E TECNOLOGIAS DE APRENDIZAGEM (LACLO 2015). CBIELACLO, 2015. Anais... CBIE-LACLO, 2015.

DEY, Niradhar. Concept and Scope of Educational Technology. Introduction to Educational Technology, Indira Gandhi National Open University (IGNOU). 2017. Disponível em: < http:// egyankosh.ac.in//handle/123456789/8409>. Acesso em: 21 jun. 2018.

HEATHER, Bellini et al. Virtual \& Augmented Reality: undertanding the race for the next computing platform. The Goldman Sachs Group, Inc. Americas: Technology. January 13, 2016. Disponível em: <http://www.goldmansachs.com/our-thinking/pages/technology-driving-innovation-folder/virtualand-augmented-reality/report.pdf > . Acesso em: 31 maio. 2017.

MISHRA, Punya; KOEHLER, Matthew J.; KERELUIK, Kristen. The song remains the same: looking back to the future of educational technology. Techtrends, [S.1.], v. 53, n. 5, September/October, 2009. 
ORGANISATION FOR ECONOMIC CO-OPERATION AND DEVELOPMENT (OECD). Education in China: a snapshot. Organisation for Economic Co-operation and Development (OECD). 2016. Disponível em: < https://www.oecd.org/china/Education-in-China-a-snapshot.pdf > . Acesso em: 21 jun. 2018.

PISA 2015 RESULTS. Excellence and Equity in Education, summarises student performance in PISA 2015, and examines inclusiveness and fairness in participating education systems. [S.l.]: OECD, 2018. Volume I. Disponível em: <http://dx.doi.org/10.1787/9789264266490-en>. Acesso em: 21 jun. 2018.

PRIOR, André. O que é tecnologia educacional. Oficina da net, 2011. Disponível em: <https:// www.oficinadanet.com.br/artigo/educacao_a_distancia/tecnologia-educacional > . Acesso em: 20 jun. 2018.

SAMSUNG. Hope for Children. Samsung solve for tomorrow. 2018. Disponível em: <http://www. samsung.com/us/corporate-citizenship/education.html>. Acesso em: 20 jun. 2018.

TIGRE, Paulo Bastos; MARQUES, Felipe Silveira. Apropriação tecnológica na economia do conhecimento: inovação e propriedade intellectual de software na América Latina. Economia e Sociedade, Campinas, v. 18, n. 3 (37), p. 547-566, dez. 2009.

WORLD INTELLECTUAL PROPERTY ORGANIZATION (WIPO). Publicação IPC. World Intellectual Property Organization. IPCPUB, v. 7, n. 2, march, 2018. Disponível em: <http://ipc.inpi. gov.br/ipcpub/?notion $=$ scheme\&version $=20180101 \&$ symbol $=$ none \&menulang $=$ pt\&lang $=$ pt\&view mode $=\mathrm{f} \&$ fipcpc $=$ no\&showdeleted $=$ yes\&indexes $=$ no\&headings $=$ yes\&notes $=$ yes\&direction $=02 \mathrm{n} \& \mathrm{i}$ nitial $=A \&$ cwid $=$ none $\&$ tree $=$ no\&searchmode $=$ smart $>$. Acesso em: 20 jun. 2018.

\section{Sobre os autores}

\section{Flávio Santos da Silva}

E-mail: flavio-santos@outlook.com

Mestrando em Propriedade Intelectual e Transferência de Tecnologia para Inovação pela Universidade Federal de Alagoas. Pós-graduado em Docência no Ensino Técnico e Profissional, Inovação e Criatividade pela Universidade Tiradentes.

Endereço profissional: Casa da Indústria Napoleão Barbosa. Avenida Fernandes Lima, n. 385, Farol, Maceió, AL. CEP: 57055-000.

\section{Leyla Carolyne da Silva Santos}

E-mail: leyla-santos@outlook.com

Mestranda em Propriedade Intelectual e Transferência de Tecnologia para Inovação pela Universidade Federal de Alagoas. Graduada em Sistemas de Informação p elo Centro Universitário Maurício de Nassau de Maceió (2016). Endereço profissional: Unidade Integrada Sesi Senai, Ebep. Avenida Antonio Lisboa de Amorim, n. 1.751, Benedito Bentes, Maceió, AL. CEP: 57084-700.

\section{Ibsen Mateus Bittencourt}

E-mail: ibsen.ead@gmail.com

Doutor em Administração de Empresas (Mackenzie-SP). Mestre Educação Brasileira na linha de Tecnologia da Informação e Comunicação (UFAL) e MBA em Gestão de Turismo (UBM-RJ).

Endereço profissional: Universidade Federal de Alagoas. Campus A. C. Simões. Avenida Lourival Melo Mota, s/n, Tabuleiro do Martins, Maceió, AL. CEP: 57072-970. 


\section{Sílvia Beatriz Uchôa}

E-mail:sbuchoa@gmail.com Doutora em Química e Biotecnologia - Área de concentração Físico-Química, pelo Instituto de Química e Biotecnologia da UFAL (2007). Mestre em Arquitetura e Planejamento, pela Escola de Engenharia de São Carlos da Universidade de São Paulo (1989).

Endereço profissional: Universidade Federal de Alagoas. Campus A. C. Simões. Avenida Lourival Melo Mota, s/n, Tabuleiro do Martins, Maceió, AL. CEP: 57072-970.

\section{Tatiane Luciano Balliano}

E-mail: tlballiano@gmail.com

Doutora em Física Aplicada (Biomolecular), pela Universidade de São Paulo (USP) (2010). Mestre em Química e Biotecnologia, área de concentração Físico-química (cristalografia de raios X) pela Universidade Federal de Alagoas (UFAL) (2006).

Endereço profissional: Universidade Federal de Alagoas, Campus A. C. Simões. Avenida Lourival Melo Mota, s/n, Tabuleiro do Martins, Maceió, AL. CEP: 57072-970. 